Journal of Molecular Genetics 2 (3-4): 41-44, 2010

ISSN: $2070-4267$

(C) Medwell Journals, 2010

\title{
Low Prevalence of Non-Subtype B HIV-1 Strains in the Texas Prisoner Population
}

\author{
${ }^{1}$ Fangling $\mathrm{Xu},{ }^{1}$ Chet Schwab, ${ }^{1}$ Xiaodong Liang, ${ }^{1}$ Scott Weaver, ${ }^{1}$ Albert Li, \\ ${ }^{2}$ Mark R. Sanborn, ${ }^{2}$ Gavin A. Cloherty and ${ }^{1}$ Jianli Dong \\ ${ }^{1}$ Department of Pathology, University of Texas Medical Branch, \\ 301 University Boulevard, TX 77555, Galveston, USA \\ ${ }^{2}$ Abbott Molecular, 1300 Touhy Ave., $\mathbb{L}$ 60018, Des Plaines, USA
}

\begin{abstract}
Genotypic drug resistance testing is standard of care in HIV management. The ViroSeq HIV-1 Genotyping System is commonly used in detecting HIV genomic mutations that confer resistance to specific types of antiretroviral drugs as an aid in treating $\mathrm{HIV}$ infection. Of note, the assay is FDA approved to detect only HIV-1 Group M Subtype B viral resistance. Subtype B was the most prevalent strain in developed counties when the assay was introduced into clinical care approximately 10 years ago. However, previously thought less common strains in the United States have gained in prevalence. The response to medications by non-B subtypes compared to $\mathrm{B}$ subtypes as well as whether the targeted mutations in non-B subtypes contribute to drug resistance the same as B subtypes need to be further studied. The purpose of this study was to identify whether HIV-1 viruses routinely tested using the ViroSeq assay in UTMB Molecular Diagnostics Laboratory are Group M Subtype B. HIV sequences generated between January and September of 2008 were aligned with $\mathrm{HIV}-1$ subtype reference strains and analyzed by phylogenetic methods. About 16 of $588 \mathrm{HIV}$ sequences $(2.7 \%)$ examined were identified as non-B subtypes including 3 of $416(0.7 \%)$ prisoner and 13 of $172(7.6 \%)$ regular patient samples.
\end{abstract}

Key words: HIV-1, subtype B, non-B subtype, ViroSeq HIV-1 Genotyping System, mutation, USA

\section{INTRODUCTION}

Human Immunodeficiency Virus (HIV) is the etiologic agent responsible for the development of Acquired Immune Deficiency Syndrome (AIDS), a chronic disease leading to immunodeficiency and susceptibility to opportunistic infections. $\mathrm{HIV}$ is a member of the genus Lentivirus belonging to the family Retroviridae. $\mathrm{HIV}$ is transmitted as single-stranded, positive-sense, enveloped RNA virus. There are two species, $H I V-1$ and $H I V-2$ that are thought to have separate introductions into human populations arising from zoonotic crossspecies transmissions from nonhuman primates to humans. HIV-1 is the cause of the great majority of HIV infections worldwide. Sequence similarity further classifies HIV-1 into groups, subtypes and sub-subtypes. Three groups of HIV-1 have been identified including $M$ (main), $\mathrm{O}$ (outlier) and $\mathrm{N}$ (non-M/non-O) (Robertson et al., 2000). The HIV-1 Group O contains a large amount of genetic diversity although the majority of infections are found in Cameroon (Roques et al., 2002). Very few isolates of the HIV-1 group $\mathrm{N}$ have been identified and sequenced. Of the three groups of $H \Pi V-1$, group $M$ dominates the global epidemic and is further classified into subtypes $\mathrm{A}, \mathrm{B}, \mathrm{C}, \mathrm{D}, \mathrm{F}, \mathrm{G}, \mathrm{H}, \mathrm{J}$ and $\mathrm{K}$. In addition, Circulating Recombinant Forms (CRFs), mosaic viruses formed between subtypes during co-or super-infection have also been recognized (http://www.hiv.lanl.gov/).

Although, subtype $\mathrm{B}$ is predominant in North America and Europe, non-B variants represent $>90 \%$ of HIV-1 circulating globally. In recently years the prevalence of non-B subtypes and CRFs in the U.S. is steadily increasing due to increased international travel and immigration (Brennan et al., 2009; Lin et al., 2006; Peeters et al., 2010). Drug resistance testing is recommended for the management of patients with HIV-1 infection (http://www.aidsinfo.nih.gov/guidelines/). The ViroSeq HIV-1 Genotyping System (Celera Diagnostics, Alameda, CA and USA): ABI PRISM 3100 genetic analyzer (Applied Biosystems, Carlsbad, CA, USA) (ViroSeq) is Food and Drug Administration (FDA) approved. The assay produces 1302 nucleotide sequences comprising codons 1-99 of the protease gene and 1-335 of the reverse transcriptase gene and is routinely used clinically in detecting mutations in the protease and Reverse Transcriptase (RT) that confer resistance to specific types of antiretroviral drugs. Although, the assay was designed specifically for HIV-1

Corresponding Author: Jianli Dong, Department of Pathology, 301 University Boulevard, Texas 77555-0743, Galveston, USA 
group $M$ subtype $B$, it can amplify and sequence HIV-1 non-B subtype sequences and has been used to provide important information about the epidemiology of HIV-1 subtypes (Brennan et al., 2009; Eshleman et al., 2004; Palma et al., 2007).

In this study it is analyzed sequences generated in the Molecular Diagnostics Laboratory at the University of Texas Medical Branch (UTMB) using the ViroSeq system for detection of HIV-1 drug resistance mutations to establish HIV-1 virus subtypes through phylogenetic analysis.

\section{MATERIALS AND METHODS}

Specimens and ViroSeq assay: The ViroSeq HIV-1 Genotyping results were generated in 588 patients referred to the Molecular Diagnostics Laboratory at UTMB between January and September of 2008. In addition to free-world patients, UTMB serves the southeast region of Texas Department of Criminal Justice units (West of Beaumont, South of Huntsville and East of San Antonio). RNA isolation, Reverse Transcription-Polymerase Chain Reaction (RT-PCR) and sequencing were performed according to the manufacturer's instructions (Yang et al., 2008). The study was approved by the UTMB Institutional Review Board (IRB).

Phylogenetic analysis: A phylogenetic tree was constructed using a total of 605 sequences including the 588 patient sequences together with homologous sequences from $17 \mathrm{HIV}-1$ subtype reference strains obtained from the Los Alamos database (http://www.hiv. lanl.gov/).

The 17 reference strains included an HIV-1 group O out-group, $13 \mathrm{HIV}-1$ group $\mathrm{M}$ subtype strains and $3 \mathrm{CRFs}$ (NCBI accession numbers: AF082395, AF084936, AF190128, AF193276, AF259955, AF286238, AJ249235, AF249238, AY169812, AY835766, FJ389367, K03454, K03455, L39106, U46016, U51190 and U54771). The sequences were aligned with ClustalW implemented in MACVECTOR version 11.1 (Accelrys, San Diego, CA and USA).

Phylogenetic trees were constructed using the Neighbor-Joining (NJ) method (18) implemented in the PAUP version 4.0a109 software package (Kumar and Gadagkar, 2000; Swofford, 2002). For NJ analysis, a distance matrix was calculated from the aligned sequences using the Hasegawa, Kishino and Yano (HK Y85) formula by allowing transitions and transversions to occur at different rates and also allowing base frequencies to vary. The phylogenetic tree was visualized with the FigTree program (http://tree.bio.ed.ac.uk). Non-B subtypes were confirmed by a second $\mathrm{NJ}$ analysis after aligning putative non-B subtypes with 17 reference sequences. The data were sampled using 1000 bootstrap replicates to determine the confidence indices within the phylogenetic tree (Felsenstein, 1988). Non-B-subtypes with bootstrap values $>80 \%$ were considered significant (Lin et al., 2006).

Statistical analysis: The Fisher's exact test was used to detect the difference in prevalence of HIV-1 non-B infection between the prison population and those in the free-world.

\section{RESULTS AND DISCUSSION}

Analysis of the sequences revealed the presence of two major clades (Fig. 1) subtype B and non-subtype B. A total of 16 of the 588 samples $(2.7 \%)$ were $H I V-1$ non-B subtypes $(10 \times \mathrm{A} / \mathrm{CRF} 01,2 \times \mathrm{C}, 1 \times \mathrm{H}, 1 \times \mathrm{D}$ and $2 \times \mathrm{CRFO} 1)$ (Fig. 2 and 3). Further analysis revealed a 10 fold difference in prevalence of non-B infection between the prison population $(0.7 \%, 3$ of 416$)$ and those in the free-world $(7.6 \%, 13$ of 172$)$; the difference is statistically significant (Fisher's Exact test, $\mathrm{p}=0.0000$ ). The significant difference in the prevalence of HIV-1 non-B infections in prisoners and free-world patients warrants further investigation. Information about the country of origin and source of HIV-1 infection should help such analysis. For example, since non-B HIV-1 strains are not prevalent in the U.S., it is possible that HIV clinics at UTMB that serve free-world patients provide care to a greater proportion of patients that were born or traveled to countries/regions that have high prevalence of non- $\mathrm{B}$

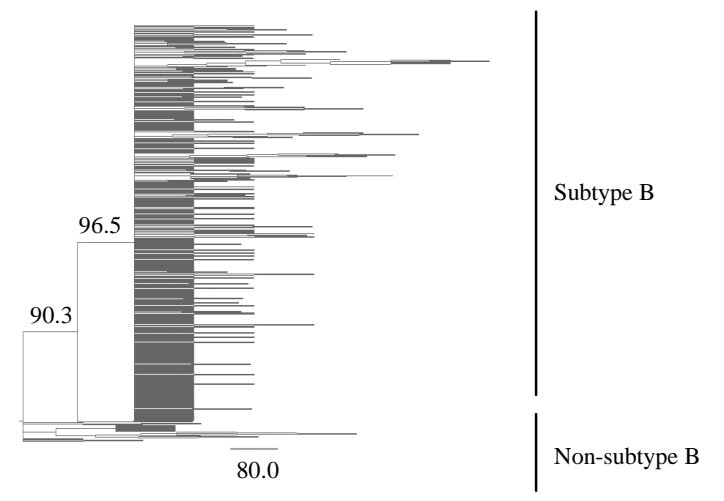

Fig. 1: Phylogenetic relationships of HIV-1 sequences based on nucleotide sequences of protease and RT regions of the Pol gene. Phylogenetic analyses were carried out using the NJ method. Distances were measured using the HKY85 formula. The bootstrap method with a NJ search was carried out with the same options. Groups with a frequency of $50 \%$ were retained. Group $\mathrm{O}$ was used as the outgroup to root the tree. Numbers adjacent to each branch represent the percentage bootstrap support calculated for 1000 replicates 


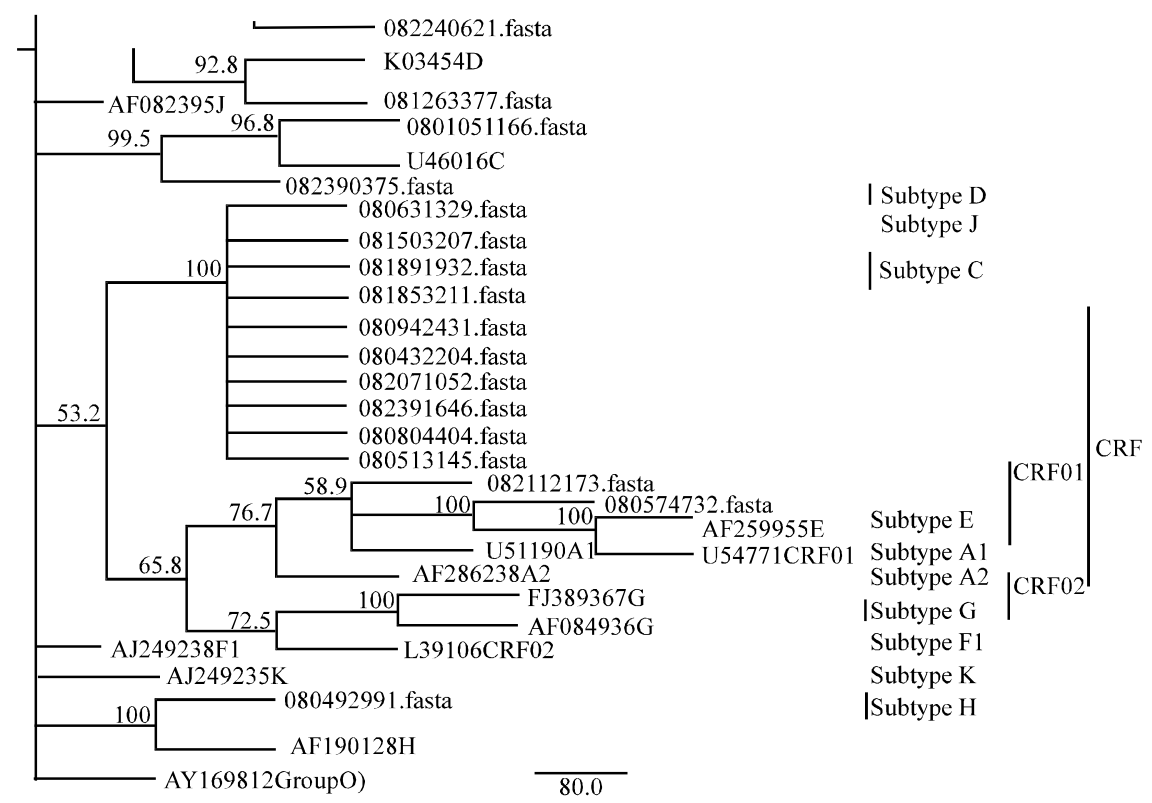

Fig. 2: Higher magnification of the NJ tree for non-subtype B sequences from Fig. 1. Numbers adjacent to each branch represent the percentage bootstrap support calculated for 1000 replicates

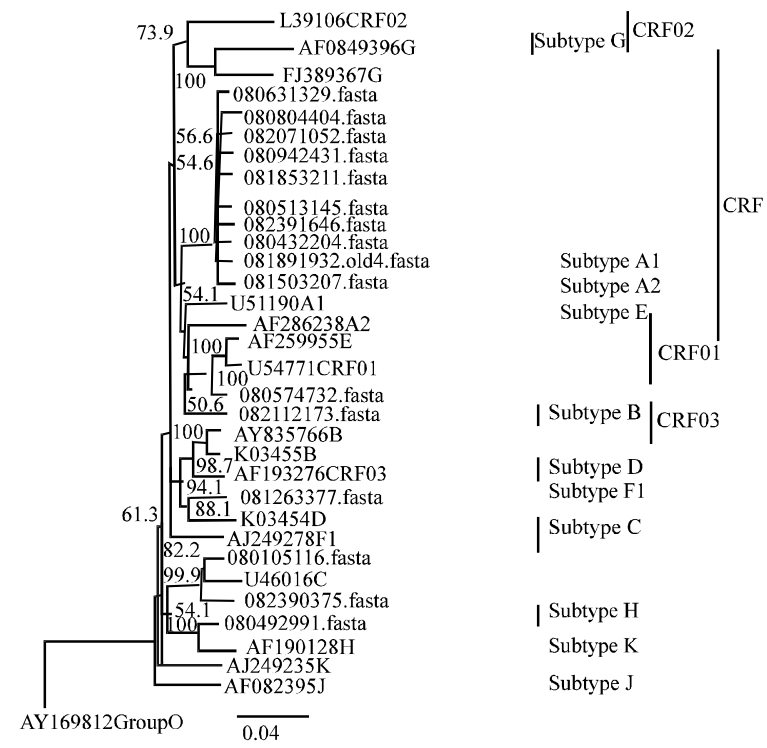

Fig. 3: Phylogenetic relationship of 16 non-subtype B sequences based on the nucleotide sequences of protease and RT regions of the Pol gene with reference sequences. Phylogenetic analyses were carried out using the NJ method. Distances were measured using the HKY85 formula. The bootstrap method with a NJ search was carried out with the same options. Groups with a frequency of $50 \%$ were retained. Group $O$ was set as the out-group to root the tree. Numbers adjacent to each branch represented the percentage bootstrap support calculated for 1000 replicates subtypes. Of note, only successfully sequenced samples were included in this study. As with other currently available molecular techniques, the performance characteristics of HIV-1 genotyping assays are subject to sequence variations existing in non-B as well as B strains. Although, there is an increasing awareness of the need to cover broader HIV strains (Church et al., 2006; Holguin et al., 2008), reagents and controls currently used in routine clinical care (including the WHO International Standard) are based on subtype B viruses. The HIV-1 ViroSeq assay was designed to identify subtype B strains. Since the assay is proprietary, the PCR and sequencing primer sequences are not available for us to perform sequence comparisons to predict annealing to various HIV-1 strains. Researchers observed sequencing failure rates of $2-8 \%$ after repeated testing.

The sequencing failure is related to viral load $(2,7$ and $8 \%$ at viral loads of $>1,000,500-999$ and 75-499 copies $\mathrm{mL}^{-1}$, respectively) with plasma input of $500 \mu \mathrm{L}$ according to the ViroSeq assay protocol (Yang et al., 2008). Viral load testing was done using the VERSANT HIV-1 RNA 3.0 Assay (bDNA, Tas in several previous publications (Church et al., 2006; Eshleman et al., 2004; Soto-Ramirez et al., 2008), this study provided another example of determining $\mathrm{HIV}-1$ subtype prevalence from resistance genotyping sequences that were routinely obtained in a clinical diagnostics laboratory. This approach is cost effective, quick and convenient to detect and monitor the HIV-1 subtype distribution and prevalence in the patient population and geographical region in which clinics/laboratories serve. Additionally, 
these sequence data are part of routine patient care; linking particular subtypes with other clinical information should help to recognize the potential impact of the growing genetic diversity of HIV-1 subtypes on patient management (Geretti, 2006; Kantor et al., 2005; Martinez-Cajas et al., 2009; Peeters et al., 2010).

\section{CONCLUSION}

The study showed that the great majority of HIV-1 infections identified in Galveston Texas were still Subtype $\mathrm{B}$ and within FDA label to perform ViroSeq HIV-1 Genotyping testing.

\section{ACKNOWLEDGEMENTS}

Researchers would like to thank members of the UTMB Molecular Diagnostics Laboratory for HIV sequencing data and Dr. David H. Walker for critical review of the manuscript.

\section{REFERENCES}

Brennan, C.A., S.L. Stramer, V. Holzmayer, J. Yamaguchi and G.A. Foster et al., 2009. Identification of human immunodeficiency virus type 1 non-B subtypes and antiretroviral drug-resistant strains in United States blood donors. Transfusion, 49: 125-133.

Church, J.D., D. Jones, T. Flys, D. Hoover and N. Marlowe et al., 2006. Sensitivity of the ViroSeq HIV-1 genotyping system for detection of the K103N resistance mutation in HIV-1 Subtypes A, C and D. J. Mol. Diagn., 8: 430-432.

Eshleman, S.H., J. Jr. Hackett, P. Swanson, S.P. Cunningham and B. Drews et al., 2004. Performance of the celera diagnostics ViroSeq HIV-1 genotyping system for sequence-based analysis of diverse human immunodeficiency virus type 1 strains. J. Clin. Microbiol., 42: 2711-2717.

Felsenstein, J., 1988. Phylogenies from molecular sequences: Inference and reliability. Ann. Rev. Genet., 22: 521-565.

Geretti, A.M., 2006. HIV-1 subtypes: Epidemiology and significance for $\mathrm{HIV}$ management. Curr. Opin. Infect. Dis., 19: 1-7.

Holguin, A., M. Lopez, M. Molinero and V. Soriano, 2008. Performance of three commercial viral load assays, Versant human immunodeficiency virus type 1 (HIV-1) RNA bDNA v3.0, Cobas AmpliPrep/Cobas TaqMan HIV-1 and NucliSens HIV-1 EasyQ v1.2, testing HIV-1 non-B subtypes and recombinant variants. J. Clin. Microbiol., 46: 2918-2923.
Kantor, R., D.A. Katzenstein, B. Efron, A.P. Carvalho and B. Wynhoven et al., 2005. Impact of HIV-1 subtype and antiretroviral therapy on protease and reverse transcriptase genotype: Results of a global collaboration. PLoS Med., 2: e112-e112.

Kumar, S. and S.R. Gadagkar, 2000. Efficiency of the neighbor-joining method in reconstructing deep and shallow evolutionary relationships in large phylogenies. J. Mol. Evol., 51: 544-553.

Lin, H.H., B.K. Gaschen, M. Collie, M. El-Fishaway and Z. Chen et al., 2006. Genetic characterization of diverse HIV-1 strains in an immigrant population living in New York City. J. Acquir. Immune. Defic. Syndr., 41: 399-404.

Martinez-Cajas, J.L., N.P. Pai, M.B. Klein and M.A. Wainberg, 2009. Differences in resistance mutations among HIV-1 non-subtype B infections: A systematic review of evidence (1996-2008). J. Int. AIDS Soc., 12: 11-11.

Palma, A.C., F. Araujo, V. Duque, F. Borges, M.T. Paixao, R. Camacho and Portuguese SPREAD Network, 2007. Molecular epidemiology and prevalence of drug resistance-associated mutations in newly diagnosed HIV-1 patients in Portugal. Infect. Genet. Evol., 7: 391-398.

Peeters, M., A.F. Aghokeng and E. Delaporte, 2010. Genetic diversity among human immunodeficiency virus-1 non-B subtypes in viral load and drug resistance assays. Clin. Microbiol. Infect., 16: 1525-1531.

Robertson, D.L., J.P. Anderson, J.A. Bradac, J.K. Carr and B. Foley et al., 2000. HIV-1 nomenclature proposal. Science, 288: 55-56.

Roques, P., D.L. Robertson, S. Souquiere, F. Damond and A. Ayouba et al., 2002. Phylogenetic analysis of 49 newly derived HIV-1 group O strains: High viral diversity but no group M-like subtype structure. Virology, 302: 259-273.

Soto-Ramirez, L.E., R. Rodriguez-Diaz, A.S. Duran, M.H. Losso and H. Salomon et al., 2008. Antiretroviral resistance among HIV type 1-infected women first exposed to antiretrovirals during pregnancy: Plasma versus PBMCs. AIDS Res. Hum. Retroviruses, 24: 797-804.

Swofford, D.L., 2002. PAUP*: Phylogenetic Analysis Using Parsimony (and Other Methods). Version 4.0 Beta, Sinauer Associates Inc., Sunderland, Massachusetts.

Yang, Z., R. Morrison, O. Oates, J. Sarria and J. Patel et al., 2008. HIV-1 genotypic resistance testing on low viral load specimens using the Abbott ViroSeq HIV-1 genotyping system. Lab. Medicine, 39: 671-673. 